\title{
Potential drug interactions during a three-decade study period: a cross-sectional study of a prescription register
}

\author{
Emelie Åstrand • Bengt Åstrand • Karolina Antonov • \\ Göran Petersson
}

Published online: 25 September 2007

(C) Springer-Verlag 2007

The cumulative incidences at the last row in Table 3, "Sum excluding: Hormonal contraceptives for systemic use Antibacterials for systemic use", should read

Table 3

\begin{tabular}{|c|c|c|c|c|c|c|c|c|}
\hline \multicolumn{3}{|c|}{ 1983-1984 } & \multicolumn{3}{|c|}{ 1993-1994 } & \multicolumn{3}{|c|}{ 2003-2004 } \\
\hline Men & Women & $\begin{array}{l}\mathrm{RR}_{\text {men/ }} \\
\text { women }\end{array}$ & Men & Women & $\begin{array}{l}\mathrm{RR}_{\text {men/ }} \\
\text { women }\end{array}$ & Men & Women & $\begin{array}{l}\mathrm{RR}_{\text {men }} \\
\text { women }\end{array}$ \\
\hline 0.108 & 0.117 & - & 0.124 & 0.125 & - & 0.108 & 0.154 & - \\
\hline
\end{tabular}

The online version of the original article can be found at http://dx.doi. org/10.1007/s00228-007-0326-0.

E. Åstrand $(\bowtie) \cdot G$. Petersson

E-Health Institute, University of Kalmar,

Bredbandet 1,

SE-392 30 Kalmar, Sweden

e-mail: emelieastrand@hotmail.com

B. Åstrand

Apoteket AB and School of Pure and Applied Natural Sciences,

University of Kalmar,

Kalmar, Sweden

K. Antonov

The Association of Pharmaceutical Industry,

Stockholm, Sweden 